\title{
Folic Acid-Functionalized Nanomedicine: Folic Acid Conjugated Copolymer and Folate Receptor Interactions Disrupt Receptor Functionality Resulting in Dual Therapeutic Anti-Cancer Potential in Breast and Prostate Cancer
}

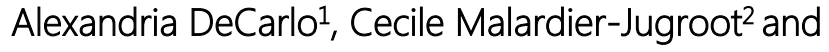 \\ Myron R. Szewczuk1, http://orcid.org/0000-0001-8471-5481 \\ 1 Department of Biomedical and Molecular Sciences, Queen's University, Kingston, ON K7L3N6, Canada. \\ 2 Department of Chemistry and Chemical Engineering, Royal Military College of Canada, Kingston, ON K7K \\ 7B4, Canada \\ * Correspondence: Myron R Szewczuk, Department of Biomedical and Molecular Sciences, Queen's University, \\ Kingston, Ontario, K7L3N6, Canada. Email: szewczuk@queensu.ca; \\ Cecile Malardier-Jugroot, Department of Chemistry and Chemical Engineering, Royal Military College of \\ Canada, Kingston, ON K7K 7B4, Canada. Email: cecile.malardier-jugroot@rmc.ca
}

Table S1. Significant $p$ values ( $n=3$, independent experiments) from Figure 4C.

\begin{tabular}{lll}
\hline & 24 Hours & 48 Hours \\
\hline Untreated vs FA-DABA-SMA & $p<0.0001$ & $p<0.0001$ \\
\hline Untreated vs anti- FR $\alpha+$ FA-DABA-SMA & $p=0.0007$ & $p=0.013$ \\
\hline Untreated vs FA + FA-DABA-SMA & $p=0.003$ & $p=0.0009$ \\
\hline Anti- FR $\alpha$ vs anti- FR $\alpha+$ FA-DABA-SMA & $\mathrm{p}<0.0001$ & $\mathrm{p}=0.004$ \\
\hline FA vs FA + FA-DABA-SMA & $\mathrm{p}=0.001$ & $\mathrm{p}=0.0021$ \\
\hline
\end{tabular}

